\title{
Differential Transforms. The Case of the Fourier Transform
}

\author{
Do Tan $\mathrm{Si}^{1}$ \\ ${ }^{1}$ HoChiMinh-city Physical Association, 40 Dong Khoi, Q1, TP.HCM, Vietnam \\ Correspondence: Do Tan Si, HoChiMinh-city Physical Association, 40 Dong Khoi, Q1, TP.HCM, Vietnam. \\ E-mail: tansi_do@yahoo.com
}

Received: March 7, 2014 Accepted: March 17, 2015 Online Published: March 24, 2015

doi:10.5539/apr.v7n2p137 URL: http://dx.doi.org/10.5539/apr.v7n2p137

\begin{abstract}
In this paper are studied the transforms of operators and functions by the exponential operators $e^{a D_{x}}, e^{a\left(\alpha D_{x}+\beta x\right)}$, $e^{a x D_{x}}, e^{a x^{2} D_{x}}, e^{\ln a \ln n x D_{x}}, e^{u(x) D_{x}}, e^{a D_{x}^{2}}, e^{a\left(\alpha D_{x}+\beta x\right)^{2}}, e^{a\left(\alpha D_{x}+\beta x\right)\left(\gamma D_{x}+\delta x\right)}, e^{i \theta\left(D_{x}^{2}+x^{2}\right)}, e^{i \theta\left(D_{x}^{2}-x^{2}\right)}$, where $D_{x} \equiv \frac{d}{d x}$ without using integration. This study is facilitated by the revelation that all relations between a couple of dual operators $(A, B)$ obeying the condition $[A, B] \equiv A B-B A \equiv I$ are invariant under substituting $(A, B)$ with any another dual couple. Compositions and decompositions of the exponential operators $e^{a\left(\alpha D_{x}+\beta x\right)}, e^{a\left(\alpha D_{x}+\beta x\right)\left(\gamma D_{x}+\delta x\right)}$ making them groups are obtained. The kernel of the integral transform associated with a differential transform is found. As case study the differential Fourier transform is highlighted in order to see how it is possible to get in a concise manner the known properties of the Fourier transform without doing integration.
\end{abstract}

Keywords: Fourier transform, fractional order Fourier transform, groups of canonical transforms of translation and dilatation, decomposition of exponential operators, kernels of integral transforms, Green's functions, transforms of geometric forms, calculus in quantum mechanics.

\section{Introduction}

The definition and properties of linear operators in a space of functions have been clearly explained by Eckart (1926) and in a space of ket vectors each defining a state of a system of particles or waves by Dirac (1930). Moreover in this book Dirac stipulates that the formulation of the laws of physics requires the use of the mathematics of transformations.

About the transformation of a function into another one by integration there are the well-known Fourier transform, the Gauss, Laplace, Mellin, Bargmann, etc...transforms, very well studied and applied (Erdélyi, Magnus, Oberhettinger, \& Tricomi, 1962, Wolf, 1979). Many noticeable references in this field from the early until recent years are given in Wolf (1979).

On the contrary although differential operators built from the "multiplication by the argument" operator $\hat{X}$ and the derivative operator $D_{x}$ (Eckart, 1926) such as the Laplacian, the Gaussian, the translation, dilatation operators, the hyperdifferential form of many canonical transforms, the Fourier transform operators, etc... are known (Liouville, 1832, Wilcox, 1967, Miller \& Steinberg, 1971, Oldham \& Spanier, 1974, Wolf, 1979, etc...) the transforms of operators by them are often not communicated and their actions onto functions are often performed via integrations like in an integral transform.

The aim of this work is to study the differential transforms, i.e. transforms by differential operators that do not use integration, by a method based on the revelation that from and only from the say canonical relation $[D, X] \equiv D X-X D \equiv I$ between a couple of say dual operators $(\mathrm{D}, \mathrm{X})$ and the unity operator I, one firstly get the fundamental relation describing the transform of the operator $\mathrm{D}$ by the operator $e^{f(X)}$

$$
e^{f(X)} D e^{-f(X)} \equiv D-f^{\prime}(X)
$$

Then, as this relation depends only on the canonical condition between the dual couple of operators $(D, X)$ we may conclude that this relation is invariant under the substitution of $(D, X)$ with any another dual couple. Moreover all the relations between operators obtained in this manner are themselves invariant under subsequent quoted substitutions. Hereinafter relations between operators will be called identities for distinguishing them with relations between functions called formulae. 
As applications it may be proved easily that the operator $e^{a D_{x}}$ is a translation operator in space and the substitutions of $\left(D_{x}, \hat{X}\right)$ with two categories of dual couples

$$
\left(\alpha D_{x}+\beta \hat{X}, \gamma D_{x}+\delta \hat{X}\right) \text { where } \alpha \delta-\beta \delta=1 \text { and }\left(\frac{1}{u^{\prime}(\hat{X})} D_{x}, u(\hat{X})\right), \forall u(x)
$$

in the relations concerning it will give rise to an infinity of operators among them are the dilatation operator, the operators that transform geometric forms, the group of operators $e^{\theta\left(\gamma \hat{X}+\delta D_{x}\right)\left(\alpha \hat{X}+\beta D_{x}\right)}$ realizing the Baker-Campbell-Hausdorff, Bargmann transforms, fractional order Fourier transform newly known in literature and the famous Fourier transform itself.

Besides, from the integral Fourier transform and the Dirac delta function we may get the transforms of operators and functions by the Gaussian transform, then by those realized by the operators $e^{a\left(\alpha D_{x}+\beta \hat{X}\right)^{2}}$.

As for cited translation and Gaussian transform, for each differential transform generated by them we will firstly calculate the transforms of the operators $D_{x}$ and $\hat{X}$, then the transform of an arbitrary function $f(x)$ and that of the Dirac delta function $\delta(x)$. The problem of decomposing a differential operator into a product of translation, dilatation and Gaussian operators is also resolved and will be utilized to obtain the kernel function of the integral transform associated with a differential transform. The compositions between exponentials of linear as so as between quadratic combinations of $D_{x}$ and $\hat{X}$ proving they are groups are performed.

In particular we will highlight the case of the differential Fourier transform in obtaining all its properties without cumbersome integrations.

\section{Background of the Method}

In a space of functions let $\mathrm{D}$ and $\mathrm{X}$ be two operators verifying the so called canonical relation

$$
D X \equiv X D+I
$$

where $\mathrm{I}$ is the unity operator defined by

$$
\text { If }(x)=f(x) \quad \forall f
$$

From the identity (1) we may deduce by recursion the following

$$
\begin{aligned}
& D^{m} X \equiv X D^{m}+m D^{m-1}, \quad \forall m \in N \\
& D^{m} X D^{-m} \equiv X+m D^{-1}, \quad \forall m \in N \\
& D^{-m} X \equiv X D^{-m}-m D^{-m-1}, \quad \forall m \in N
\end{aligned}
$$

In fact $(2 \mathrm{a})$ and $(2 \mathrm{~b})$ are valuable for $\mathrm{m}$ rational as we can see when putting $Y^{n} \equiv D^{m}$ in (2a) and calculating the operator $A(D)$ such that $Y X \equiv X Y+A(D)$. Moreover as a real number is the common limit of two sequences of rational numbers we may assert that $(2 \mathrm{a})$ and $(2 \mathrm{~b})$ are valuable for $\mathrm{m}$ real.

Now let $f(x)$ be a function and $f^{\prime}(x)$ its derivative function

$$
\begin{aligned}
& f(x)=\sum_{m} a_{m} x^{m}, m \in R \\
& f^{\prime}(x)=\sum_{m} a_{m} m x^{m-1}
\end{aligned}
$$

we get from (2) the identity

$$
f(D) X \equiv X f(D)+f^{\prime}(D)
$$

i.e.

$$
[f(D), X] \equiv f(D) X-X f(D) \equiv f^{\prime}(D)
$$

where the notation $[A, B]$ designates the so called commutator between $\mathrm{A}$ and $\mathrm{B}$. 
Because (3a) is obtained from and only from (1) we may state:

"From and only from the relation $[D, X] \equiv I$ between two operators $\mathrm{D}$ and X happens the identity $[f(D), X] \equiv f^{\prime}(D)$ where $\mathrm{f}(\mathrm{x})$ is any derivable function. This identity per consequence is invariant under the substitution of the couple of operators $(D, X)$ with any another couple $\left(D^{\prime}, X^{\prime}\right)$ provided that $\left[D^{\prime}, X^{\prime}\right] \equiv I$. Moreover, all the identities between operators created from (3a) by such substitutions are also invariant under subsequent ones“. Remark that in the above statement $\left(D^{\prime}, X^{\prime}\right)$ is not only the couple $(\alpha D+\beta X, \gamma D+\delta X)$ where $\alpha \delta-\beta \gamma=1$ as in a canonical transform but may be the couple of operators such as $\left(-X^{2} D, X^{-1}\right)$ as we can see later.

For first example, by substituting $(D, X)$ with $(X,-D)$ we get

$$
[D, f(X)] \equiv f^{\prime}(X)
$$

The identities (3a), (3b) lead to the more familiar ones

$$
\begin{gathered}
e^{-f(X)} D e^{f(X)} \equiv D+f^{\prime}(X) \\
e^{f(D)} X e^{-f(D)} \equiv X+f^{\prime}(D)
\end{gathered}
$$

which give the transform of $\mathrm{D}$ by $e^{-f(X)}$ and of $\mathrm{X}$ by $e^{f(D)}$.

In particular

$$
\begin{aligned}
& e^{-a X} D e^{a X} \equiv D+a \\
& e^{a D} X e^{-a D} \equiv X+a
\end{aligned}
$$

Applying n times (5a), (5b) we get the transforms of $D^{n}$ by $e^{-a X}$ and of $X^{n}$ by $e^{a D}$.

By the same manner we see that

$$
\begin{aligned}
& e^{-a X^{2}} D e^{a X^{2}} \equiv D+2 a X \\
& e^{a D^{2}} X e^{-a D^{2}} \equiv 2 a D+X
\end{aligned}
$$

so that, for $f(x)$ derivable, $e^{-a X^{2}}$ transforms an operator $f(D)$ into $f(D+2 a X)$ and $e^{a D^{2}}$ an operator $f(X)$ into $f(X+2 a D)$.

The meaning of the above identities is that one can factorize $f(D+a)$ or $f(a D+b X)$ into a product of three simple operators.

In order to generalize the identities (5a), (5b) we substitute $(D, X)$ with $(\alpha D+\beta X, \gamma D+\delta X)$ where $(\alpha \delta-\beta \gamma)=1$ and get

$$
\begin{aligned}
& e^{a(\alpha D+\beta X)}(\alpha D+\beta X) e^{-a(\alpha D+\beta X)} \equiv(\alpha D+\beta X) \\
& e^{a(\alpha D+\beta \hat{X})}(\gamma D+\delta X) e^{-a(\alpha D+\beta X)} \equiv(\gamma D+\delta X)+\alpha a I
\end{aligned}
$$

i.e.

$$
\begin{aligned}
& e^{a(\alpha D+\beta X)} D e^{-a(\alpha D+\beta X)} \equiv D-\beta a I \\
& e^{a(\alpha D+\beta X)} X e^{-a(\alpha D+\beta X)} \equiv X+\alpha a I
\end{aligned}
$$

Utilizing (6a) then (5b), we get the identity which governs the decomposition of $e^{a(\alpha D+\beta X)}$ 


$$
\begin{aligned}
e^{a(\alpha D+\beta X)} \equiv e^{-\frac{\beta}{2 \alpha} X^{2}} e^{a \alpha D_{x}} e^{\frac{\beta}{2 \alpha} X^{2}} & \equiv e^{-\frac{\beta}{2 \alpha} X^{2}} e^{a \alpha D} e^{\frac{\beta}{2 \alpha} X^{2}} e^{-a \alpha D} e^{a \alpha D} \\
& \equiv e^{-\frac{\beta}{2 \alpha} X^{2}} e^{\frac{\beta}{2 \alpha}(X+a \alpha I)^{2}} e^{a \alpha D} \equiv e^{\frac{1}{2} \alpha \beta a^{2}} e^{\beta a X} e^{a \alpha D}
\end{aligned}
$$

Substituting $(D, X)$ with $(-X, D)$ we get another mode of decomposition for $e^{a(\alpha D+\beta X)}$

$$
e^{a(\alpha D+\beta X)} \equiv e^{-\frac{1}{2} \alpha \beta a^{2}} e^{a \alpha D} e^{a \beta X}
$$

From (5b) and (8b) we get

$$
e^{\left(\alpha^{\prime} D+\beta^{\prime} X\right)} e^{(\alpha D+\beta X)} \equiv e^{\frac{1}{2}\left(\alpha \beta+\alpha^{\prime} \beta^{\prime}\right)} e^{\beta^{\prime} X+\beta\left(X+\alpha^{\prime}\right)} e^{\left(\alpha+\alpha^{\prime}\right) D}=e^{\frac{1}{2}\left(\alpha^{\prime} \beta-\alpha \beta^{\prime}\right)} e^{\left(\alpha+\alpha^{\prime}\right) D+\left(\beta^{\prime}+\beta\right) X}
$$

and may conclude that the differential transforms realized by the operators $e^{\alpha D+\beta X}$ form a group.

Hereinafter we will utilize essentially (8a) to get the kernel of an integral transform associated with a differential one.

\section{The Differential Transforms}

\subsection{Translation, Group of Transforms by $e^{\alpha D_{x}+\beta \hat{X}}$, Kernel of Integral Transforms}

Let $D_{x}$ be the derivative operator and $\hat{X}$ the "multiplication by the argument $\mathrm{x}$ " operator in the space of differentiable functions (Eckart, 1926)

$$
\begin{aligned}
& D_{x} f(x)=f^{\prime}(x) \quad \forall f \\
& \hat{X} f(x)=x f(x) \quad \forall f
\end{aligned}
$$

Because

$$
D_{x} \hat{X} f(x)=(x f(x))^{\prime}=x f^{\prime}(x)+f(x) \quad \forall f \text { differentiable }
$$

we have

$$
D_{x} \hat{X} \equiv \hat{X} D_{x}+I
$$

so that according to $(5 \mathrm{~b})$

$$
e^{a D_{x}} f(\hat{X}) e^{-a D_{x}} \equiv f(\hat{X}+a I)
$$

Acting the above identity onto a constant we get the formula

$$
e^{a D_{x}} f(x)=f(x+a)
$$

which means that $e^{a D_{x}}$ realized the translation in a one dimensional space.

By substitution of $\left(D_{x}, \hat{X}\right)$ with $\left(\alpha D_{x}+\beta \hat{X}, \gamma D_{x}+\delta \hat{X}\right)$ where $(\alpha \delta-\beta \gamma)=1$ in (7a), (7b) we get the transforms by $e^{a\left(\alpha D_{x}+\beta \hat{X}\right)}$ of $D_{x}$ and $\hat{X}$

$$
\begin{aligned}
& e^{a\left(\alpha D_{x}+\beta \hat{X}\right)} D_{x} e^{-a\left(\alpha D_{x}+\beta \hat{X}\right)} \equiv D_{x}-\beta a I \\
& e^{a\left(\alpha D_{x}+\beta \hat{X}\right)} \hat{X} e^{-a\left(\alpha D_{x}+\beta \hat{X}\right)} \equiv \hat{X}+\alpha a I
\end{aligned}
$$

and from (8a) the formula

$$
\begin{aligned}
& e^{a\left(\alpha D_{x}+\beta \hat{X}\right)} \equiv e^{\frac{1}{2} \alpha \beta a^{2}} e^{a \beta \hat{X}} e^{a \alpha D_{x}} \\
& e^{\alpha D_{x}+\beta \hat{X}} f(x)=e^{\frac{1}{2} \alpha \beta} e^{\beta x} f(x+\alpha)
\end{aligned}
$$

Formula (11d) may be useful in quantum mechanics, for example 


$$
e^{\lambda a^{+}} f(x)=e^{\lambda\left(\frac{D_{x}+\hat{X}}{\sqrt{2}}\right)} f(x)=e^{\frac{1}{4} \lambda^{2}} e^{\frac{\lambda}{\sqrt{2}} x} f\left(x+\frac{\lambda}{\sqrt{2}}\right)
$$

Now, let $A\left(D_{x}, \hat{X}\right)$ be an operator which transforms $D_{x}$ into $\left(\alpha D_{x}+\beta \hat{X}\right)$ and $\hat{X}$ into $\left(\gamma D_{x}+\delta \hat{X}\right)$

with $\alpha \delta-\beta \gamma=1$ we have

$$
A f\left(x-x_{0}\right)=A e^{-x_{0} D_{x}} A^{-1} A f(x)=e^{-x_{0}\left(\alpha D_{x}+\beta \hat{X}\right)} A f(x)=e^{-\frac{1}{2} x_{0}^{2} \alpha \beta} e^{-x_{0} \beta \hat{X}} e^{-x_{0} \alpha D_{x}} A f(x)
$$

Using a property of the Dirac delta function we may write

$$
A\left(D_{x}, \hat{X}\right) f(x)=A\left(D_{x}, \hat{X}\right) \int_{-\infty}^{+\infty} f\left(x_{0}\right) \delta\left(x-x_{0}\right) d x_{0} .
$$

and see that the kernel function of the integral transform linked to the differential transform realized by $A\left(D_{x}, \hat{X}\right)$ may be obtained if we can calculate $A\left(D_{x}, \hat{X}\right) \delta(x)$ according to (11a), (11c) as follows

$$
\begin{aligned}
K\left(x, x_{0}\right) & =A\left(D_{x}, \hat{X}\right) \delta\left(x-x_{0}\right)=A\left(D_{x}, \hat{X}\right) e^{-x_{0} D_{x}} \delta(x)=e^{-x_{0}\left(\alpha D_{x}+\beta D_{x}\right)} A\left(D_{x}, \hat{X}\right) \delta(x) \\
& =e^{\frac{1}{2} x_{0}^{2} \alpha \beta} e^{-x_{0} \beta \hat{X}} e^{-x_{0} \alpha D_{x}} A\left(D_{x}, \hat{X}\right) \delta(x)
\end{aligned}
$$

Inversely knowing $K\left(x, x_{0}\right)$ we may calculate

$$
A\left(D_{x}, \hat{X}\right) \delta(x)=e^{\frac{1}{2} x_{0}^{2} \alpha \beta} e^{x_{0} \alpha D_{x}} e^{x_{0} \beta \hat{X}} K\left(x, x_{0}\right)
$$

and obtain $\alpha, \beta$ by demanding the second member to be independent with respect to $x_{0}$. Then as

$$
A\left(D_{x}, \hat{X}\right) \hat{X} A^{-1}\left(D_{x}, \hat{X}\right) \equiv\left(\gamma D_{x}+\delta \hat{X}\right) \Rightarrow\left(\gamma D_{x}+\delta \hat{X}\right) A\left(D_{x}, \hat{X}\right) \delta(x)=0
$$

we may obtain $\gamma, \delta$ if the second member of (12d) is a Gaussian function or a constant.

\subsection{The Dilatation Operator $e^{a \hat{X} D_{x}}$}

Because

$$
\left[\hat{X} D_{x}, \ln \hat{X}\right] \equiv \hat{X}\left[D_{x}, \ln \hat{X}\right]+[\hat{X}, \ln \hat{X}] D_{x} \equiv I
$$

we may substitute the couple $\left(D_{x}, \hat{X}\right)$ with $\left(\hat{X} D_{x}, \ln \hat{X}\right)$ in the translation identity (7a)

$$
e^{a D_{x}} \hat{X} e^{a D_{x}} \equiv \hat{X}+a I
$$

in order to get

$$
e^{a \hat{X} D_{x}} \ln \hat{X} e^{-a \hat{X} D_{x}} \equiv \ln \hat{X}+a
$$

and, successively,

$$
\begin{aligned}
& e^{a \hat{X} D_{x}} e^{\ln \hat{X}} e^{-a \hat{X} D_{x}} \equiv e^{\ln \hat{X}+a} \equiv e^{a} \hat{X} \\
& e^{a \hat{X} D_{x}} \hat{X} e^{-a \hat{X} D_{x}} \equiv e^{a} \hat{X}
\end{aligned}
$$

Substituting $\left(D_{x}, \hat{X}\right)$ with $\left(-\hat{X}, D_{x}\right)$ in (13a) we also have

$$
e^{a \hat{X} D_{x}} D_{x} e^{-a \hat{X} D_{x}} \equiv e^{-a} D_{x}
$$

From (13a) we may get the formulae

$$
e^{a \hat{X} D_{x}} f(x)=f\left(e^{a} x\right)
$$




$$
\begin{aligned}
& e^{a \hat{X} D_{x}} \delta(x)=\delta\left(e^{a} x\right)=e^{-a} \delta(x) \\
& e^{a \hat{X} D_{x}} \delta\left(x-x_{0}\right)=e^{a \hat{X} D_{x}} e^{-x_{0} D_{x}} \delta(x)=e^{-x_{0} e^{-a} D_{x}} e^{a \hat{X} D_{x}} \delta(x)=\left|e^{-a}\right| \delta\left(x-x_{0} e^{-a}\right)
\end{aligned}
$$

Formula (13c) means that $e^{a \hat{X} D_{x}}$ is the operator describing a dilatation. The kernel function related to it is given by (13e).

Viewing the way $e^{a \hat{X} D_{x}}$ is obtained we may say that a dilatation $e^{a\left(\hat{X} D_{x}+\hat{Y} D_{y}+\hat{Z} D_{z}\right)}$ is in fact a translation in a space where the coordinates are rescaled from $\mathrm{x}, \mathrm{y}, \mathrm{z}$ down to $\ln \mathrm{x}, \ln \mathrm{y}, \ln \mathrm{z}$.

\subsection{The Hyperbolic Transform $e^{-a \hat{X}^{2} D_{x}}$}

From the fact that

$$
\left[-\hat{X}^{2} D_{x}, \hat{X}^{-1}\right] \equiv-\hat{X}^{2}\left[D_{x}, \hat{X}^{-1}\right]-\left[\hat{X}^{2}, \hat{X}^{-1}\right] D_{x} \equiv I
$$

we may substitute $\left(D_{x}, \hat{X}\right)$ with $\left(-\hat{X}^{2} D_{x}, \hat{X}^{-1}\right)$ in the translation identities (7a), (7b) and obtain successively

$$
\begin{aligned}
& e^{-a \hat{X}^{2} D_{x}} \hat{X}^{-1} e^{a \hat{X}^{2} D_{x}} \equiv \hat{X}^{-1}+a I \\
& e^{-a \hat{X}^{2} D_{x}} \hat{X} e^{a \hat{X}^{2} D_{x}} \equiv\left(\hat{X}^{-1}+a I\right)^{-1} \\
& e^{-a \hat{X}^{2} D_{x}} \hat{X}^{2} D_{x} e^{a \hat{X}^{2} D_{x}} \equiv \hat{X}^{2} D_{x} \\
& e^{-a \hat{X}^{2} D_{x}} D_{x} e^{a \hat{X}^{2} D_{x}} \equiv\left(\hat{X}^{-1}+a I\right)^{2} \hat{X}^{2} D_{x} \equiv(I+a \hat{X})^{2} D_{x} \\
& e^{-a \hat{X}^{2} D_{x}} f(x)=f\left(\frac{x}{1+a x}\right) \\
& e^{-a \hat{X}^{2} D_{x}} \delta\left(x-x_{0}\right)=e^{-a \hat{X}^{2} D_{x}} e^{-x_{0} D_{x}} f(x)=e^{-x_{0}(I+a \hat{X}) D_{x}} \delta\left(\frac{x}{1+a x}\right)=e^{\frac{1}{a} D_{x}} e^{-x_{0} a \hat{X}} D_{x} e^{-\frac{1}{b} D_{x}} \delta\left(\frac{x}{1+a x}\right) \\
& \quad=e^{\frac{1}{a} D_{x}} e^{-x_{0} a \hat{X}^{2} D_{x}} \delta\left(\frac{a x-1}{a^{2} x}\right)=\delta\left(\frac{x}{1+a x}-x_{0}\right)
\end{aligned}
$$

From (15d) we get the kernel function of the integral transform related to $e^{-a \hat{X}^{2} D_{x}}$. From (15a) we see that $e^{-a(\hat{X}+b)^{2} D_{x}}$ transforms the straight line $y=x$ into the hyperbola $y=\frac{(1-a b) x-a b^{2}}{a x+(1+a b)}$.

3.4 The Transforms of Geometric Forms $e^{\ln a \hat{X} \ln \hat{X} D_{x}+\ln b \hat{Y} \ln \hat{Y} D_{y}+\ln c \hat{Z} \ln \hat{Z} D_{z}}$

Because

$$
\left[\hat{X} \ln \hat{X} D_{x}, \ln \ln \hat{X}\right] \equiv \hat{X} \ln \hat{X}\left[D_{x}, \ln \ln \hat{X}\right]+[\hat{X} \ln \hat{X}, \ln \ln \hat{X}] D_{x} \equiv I
$$

we may substitute $\left(D_{x}, \hat{X}\right)$ with $\left(\hat{X} \ln \hat{X} D_{x}, \ln \ln \hat{X}\right)$ in (7a), (7b) in order to obtain successively

$$
\begin{aligned}
& e^{\ln a \hat{X} \ln \hat{X} D_{x}} \ln \ln \hat{X} e^{-\ln a \hat{X} \ln \hat{X} D_{x}} \equiv \ln \ln \hat{X}+\ln a, a>0 \\
& e^{\ln a \hat{X} \ln \hat{X} D_{x}} \ln \hat{X} e^{-\ln a \hat{X} \ln \hat{X} D_{x}} \equiv a \ln \hat{X} \\
& e^{\ln a \hat{X} \ln \hat{X} D_{x}} \hat{X} e^{-\ln a \hat{X} \ln \hat{X} D_{x}} \equiv \hat{X}^{a} \\
& e^{\ln a \hat{X} \ln \hat{X} D_{x}} D_{x} e^{-\ln a \hat{X} \ln \hat{X} D_{x}} \equiv a^{-1} \hat{X}^{1-a} D_{x}
\end{aligned}
$$

From (17a) we get the transform of a function 


$$
\begin{aligned}
& e^{\ln a \hat{X} \ln \hat{X} D_{x}} f(x)=f\left(x^{a}\right), \mathrm{a}>0, \mathrm{x}>0 \\
& e^{\ln a \hat{X} \ln \hat{X} D_{x}} f(-x)=f\left((-x)^{a}\right), \mathrm{a}>0, \mathrm{x}<0
\end{aligned}
$$

Because $\left[a^{-1} \hat{X}^{1-a} D_{x}, \hat{X}^{a}\right] \equiv I$ we have

$$
e^{-x_{0} a^{-1} \hat{X}^{1-a} D_{x}} x^{a}=x^{a}-x_{0}
$$

so that, for a $>0$

$$
\begin{aligned}
& e^{\ln a \hat{X} \ln \hat{X} D_{x}} f\left(x-x_{0}\right)=e^{\ln a \hat{X} \ln \hat{X} D_{x}} e^{-x_{0} D_{x}} f(x)=e^{-x_{0} a^{-1} \hat{X}^{1-a} D_{x}} f\left(x^{a}\right)=f\left(x^{a}-x_{0}\right), \quad \mathrm{x}>0 \\
& e^{\ln a \hat{X} \ln \hat{X} D_{x}} f\left(-x-x_{0}\right)=f\left((-x)^{a}-x_{0}\right), \quad \mathrm{x}<0 \\
& e^{\ln a \hat{X} \ln \hat{X} D_{x}} \delta\left(x-x_{0}\right)=\delta\left((-x)^{a}+x_{0}\right), \quad \mathrm{x}<0
\end{aligned}
$$

From (17d) we may get the kernel function of the integral transform related to $e^{\ln a \hat{X} \ln \hat{X} D_{x}}$.

$$
\begin{aligned}
& K\left(x, x_{0}\right)=\delta\left(x^{a}-x_{0}\right), \quad \mathrm{x}>0 \\
& K\left(x, x_{0}\right)=\delta\left((-x)^{a}+x_{0}\right), \quad \mathrm{x}<0
\end{aligned}
$$

A particular case is

$$
\begin{array}{ll}
e^{\ln 2 \hat{X} \ln \hat{X} D_{x}} x=x^{2} & \forall x>0 \\
e^{\ln 2 \hat{X} \ln \hat{X} D_{x}}(-x)=x^{2} & \forall x<0
\end{array}
$$

so that

$$
e^{\ln 2 \hat{X} \ln \hat{X} D_{x}} x=x|x| \quad \forall x
$$

and the double pyramid

$$
|x|+|y|+|z|=1
$$

is transformed by $e^{\ln 2 \hat{X} \ln \hat{X} D_{x}} e^{\ln 2 \hat{Y} \ln \hat{Y} D_{y}} e^{\ln 2 \hat{Z} \ln \hat{Z} D_{z}}$ into the sphere

$$
x^{2}+y^{2}+z^{2}=1
$$

In this transformation a point $\left(x_{0}, y_{0}, z_{0}\right)$ on the double pyramid is transformed into the point $\left(\frac{x_{0}}{\sqrt{\left|x_{0}\right|}}, \frac{y_{0}}{\sqrt{\left|y_{0}\right|}}, \frac{z_{0}}{\sqrt{\left|z_{0}\right|}}\right)$

situated on the sphere. Moreover the square $|x|+|y|+z_{0}=1$ on the upper pyramid is transformed into a circle intersection between the sphere and the plane $z=\sqrt{z_{0}}$.

Another interesting example is that the plane

$$
x+y+z=1
$$

is transformed into a part of the sphere in the subspace $(+++)$ where $x, y, z$ are all positive and a part of a hyperboloid $\left(x^{2}+y^{2}-z^{2}\right)=1$ in the subspace (++-) where $\mathrm{x}, \mathrm{y}$ are positive although $\mathrm{z}$ is negative and so on. We see also that a straight line in this plane is transformed into an arc of circle in the subspace $(+++)$ jointed with six arcs of hyperbola in other subspaces excepted the (---). 


\subsection{The Transforms by $e^{g(\hat{X}) D_{x}}$}

Let

$$
u(x)=\int \frac{1}{g(x)}
$$

we have

$$
\left[g(\hat{X}) D_{x}, u(\hat{X})\right] \equiv g(\hat{X})\left[D_{x}, u(\hat{X})\right] \equiv g(\hat{X}) u^{\prime}(\hat{X}) \equiv I
$$

so that by substitution of $\left(D_{x}, \hat{X}\right)$ with $\left(g(\hat{X}) D_{x}, u(\hat{X})\right)$ in the translation identities we get

$$
\begin{aligned}
& e^{a g(\hat{X}) D_{x}} u(\hat{X}) e^{-a g(\hat{X}) D_{x}} \equiv u(\hat{X})+a I \\
& e^{a g(\hat{X}) D_{x}} g(\hat{X}) D_{x} e^{-a g(\hat{X}) D_{x}} \equiv g(\hat{X}) D_{x} \\
& e^{a g(\hat{X}) D_{x}} g(\hat{X}) D_{x} e^{-a g(\hat{X}) D_{x}} \equiv g(\hat{X}) D_{x} \\
& e^{a g(\hat{X}) D_{x}} \hat{X} e^{-a g(\hat{X}) D_{x}} \equiv u^{-1}(u(\hat{X})+a I), \text { with } u^{-1}(u(x))=x \\
& e^{a g(\hat{X}) D_{x}} D_{x} e^{-a g(\hat{X}) D_{x}} \equiv \frac{1}{g\left(u^{-1}(u(\hat{X})+a I)\right)} g(\hat{X}) D_{x} \\
& e^{a g(x) D_{x}} f(x)=f\left(u^{-1}(u(x)+a)\right) \\
& e^{a g(x) D_{x}} f\left(x-x_{0}\right)=e^{a g(x) D_{x}} e^{-x_{0} D_{x}} f(x)=e^{-x_{0} \frac{g(\hat{X})}{g\left(u^{-1}(u(\hat{X})+a I)\right)} D_{x}} e^{a g(x) D_{x}} f(x)
\end{aligned}
$$

The transform of $\delta(x)$ may be obtained from (20c) and the formula (23d) due to Wolf (Wolf 1979).

For example, for $g(x)=\cos x$ we get

$$
\begin{aligned}
& u=\int \frac{1}{\cos x}=\int \frac{\cos x}{1-\sin ^{2} x}=\frac{1}{2} \ln \frac{1+\sin x}{1-\sin x} \\
& e^{a \cos \hat{X} D_{x}} \ln \frac{1+\sin \hat{X}}{1-\sin \hat{X}} e^{-a \cos \hat{X} D_{x}} \equiv \ln \frac{1+\sin \hat{X}}{1-\sin \hat{X}}+2 a \\
& e^{a \cos \hat{X} D_{x}} \sin \hat{X} e^{-a \cos \hat{X} D_{x}} \equiv \frac{\left(e^{2 a}+1\right) \sin \hat{X}+\left(e^{2 a}-1\right)}{\left(e^{2 a}-1\right) \sin \hat{X}+\left(e^{2 a}+1\right)} \\
& e^{a \cos \hat{X} D_{x}} f(\hat{X}) e^{-a \cos \hat{X} D_{x}} \equiv f\left(\arcsin \frac{\left(e^{2 a}+1\right) \sin \hat{X}+\left(e^{2 a}-1\right)}{\left(e^{2 a}-1\right) \sin \hat{X}+\left(e^{2 a}+1\right)}\right)
\end{aligned}
$$

Act the above identity onto a constant we get

$$
e^{a \cos x D_{x}} f(x)=f\left(\arcsin \frac{\left(e^{2 a}+1\right) \sin x+\left(e^{2 a}-1\right)}{\left(e^{2 a}-1\right) \sin x+\left(e^{2 a}+1\right)}\right)
$$

We see that the transforms of $\sin x, \cos x=\sqrt{1-\sin x}$ then of $\sin p x, \cos p x$ via the de Moivre formula may be calculated straightforwardly.

\subsection{The Gaussian Transform and Its Generalization}

According to (6a) and (6b) we get

$$
e^{-a \hat{X}^{2}} D_{x} e^{a \hat{X}^{2}} \equiv D_{x}+2 a \hat{X}
$$




$$
e^{-a D_{x}^{2}} \hat{X} e^{a D_{x}^{2}} \equiv \hat{X}-2 a D_{x}
$$

In order to calculate the transform by $e^{a D_{x}^{2}}$ of the Gaussian $e^{b x^{2}}$ let us begin with the conventional definition of the Fourier transform (Wolf 1979) denoted hereinafter by the notation FT

$$
\operatorname{FTf}(x)=\frac{1}{\sqrt{2 \pi}} \int_{-\infty}^{+\infty} e^{-i x x_{0}} f\left(x_{0}\right) d x_{0}
$$

together with that of the Dirac delta function $\delta(x)$ (Dirac, 1930)

$$
\delta(x)=2 \pi \int_{-\infty}^{+\infty} e^{-i x x_{0}} d x_{0}
$$

which has the properties

$$
\begin{aligned}
& x \delta(x)=0 \\
& \int_{-\infty}^{+\infty} \delta(x) d x=1 \\
& \delta(f(x))=\sum_{i}\left|f^{\prime}\left(a_{i}\right)\right|^{-1} \delta\left(x-a_{i}\right) \text { where } f\left(a_{i}\right)=0, f^{\prime}\left(a_{i}\right) \neq 0 \\
& f(x)=\int_{-\infty}^{+\infty} f\left(x_{0}\right) \delta\left(x-x_{0}\right) d x_{0}
\end{aligned}
$$

From the above definitions we get for $a>0$ or $a=i \theta$

$$
\begin{aligned}
& F T 1=\sqrt{2 \pi} \delta(x) \\
& F T e^{-a x^{2}}=(2 a)^{-\frac{1}{2}} e^{-x^{2} / 4 a} \\
& F T e^{-b D_{x}^{2}} e^{-a x^{2}}=e^{b x^{2}} F T e^{-a x^{2}}=(2 a)^{-\frac{1}{2}} e^{-\frac{1-4 a b}{4 a} x^{2}} \\
& F T e^{-a D_{x}^{2}} e^{-\frac{1}{4 a} x^{2}}=e^{a x^{2}} F T e^{-\frac{1}{4 a} x^{2}}=e^{a x^{2}} \sqrt{2 a} e^{-a x^{2}}=\sqrt{2 a}
\end{aligned}
$$

so that the transforms of the Gaussian and the Dirac delta functions $\delta(x)$ are given by the formulae

$$
\begin{aligned}
& e^{-b D_{x}^{2}} e^{-a x^{2}}=(1-4 a b)^{-\frac{1}{2}} e^{-\frac{a}{1-4 a b} x^{2}},(1-4 a b)>0 \text { if a real } \\
& e^{-a D_{x}^{2}} e^{-\frac{1}{4 a^{2}}}=F T \sqrt{2 a}=\sqrt{4 \pi a} \delta(x) \quad a>0 \text { or } a=i \theta
\end{aligned}
$$

In order to obtain the transform by $e^{a D_{x}{ }^{2}}$ of a function $f(x)$ let us consider the differential equation

$$
\left(D_{x}^{2}-2 x D_{x}+2 n\right) y=0
$$

of which a particular solution is known as the Hermite polynomial $H_{n}(x)$ of order $\mathrm{n}$ (Abramowitz \& Stegun, 1968). According to (21b) we get

$$
\begin{aligned}
e^{\frac{1}{4} D_{x}^{2}}\left(D_{x}^{2}-2 x D_{x}+2 n\right) e^{-\frac{1}{4} D_{x}^{2}} e^{\frac{1}{4} D_{x}^{2}} y & =\left(D_{x}^{2}-\left(2 x+D_{x}\right) D_{x}+2 n\right) e^{\frac{1}{4} D_{x}^{2}} y \\
& =\left(2 x D_{x}-2 n\right) e^{\frac{1}{4} D_{x}^{2}} y=0
\end{aligned}
$$

so that

$$
e^{\frac{1}{4} D_{x}^{2}} y=H_{n}(x)=c_{n} x^{n}
$$


As the Hermite polynomials verify the recursion relation

$$
H^{\prime}{ }_{n}(x)=2 n H_{n-1}(x)
$$

we get finally the interesting formula

$$
H_{n}(x)=e^{-D^{2} / 4}(2 x)^{n}
$$

which has been known (Wolf 1976 ) and is easier to handle than the Rodrigues one.

From (30) we get the transform by $e^{-a D_{x}^{2}}$ of $x^{n}$

$$
e^{-a D_{x}^{2}}\left(\frac{x}{\sqrt{a}}\right)^{n}=H_{n}\left(\frac{x}{2 \sqrt{a}}\right)
$$

From (31) we may obtain the transform by $e^{-a D_{x}^{2}}$ of a function $f(x)$ expandable into Taylor series under the form of a series of Hermite polynomials.

Now by substituting $\left(D_{x}, \hat{X}\right)$ with $\left(\alpha D_{x}+\beta \hat{X}, \gamma D_{x}+\delta \hat{X}\right)$ where $(\alpha \delta-\beta \gamma)=1$ in (21a), (21 b) we get

$$
\begin{aligned}
& \left.e^{-a\left(\gamma D_{x}+\delta \hat{X}\right)^{2}} D_{x} e^{a\left(\gamma D_{x}+\delta \hat{X}\right)^{2}} \equiv(1+2 a \gamma \delta) D_{x}+2 a \delta^{2} \hat{X}\right) \\
& e^{-a\left(\gamma D_{x}+\delta \hat{X}\right)^{2}} \hat{X} e^{a\left(\gamma D_{x}+\delta \hat{X}\right)^{2}} \equiv-2 a \gamma^{2} D_{x}+(1-2 a \gamma \delta) \hat{X}
\end{aligned}
$$

From (21a), (21b) also we may write

$$
\gamma D_{x}+\delta \hat{X} \equiv e^{\frac{\gamma}{2 \delta} D_{x}^{2}} \delta \hat{X} e^{-\frac{\gamma}{2 \delta} D_{x}^{2}} \equiv e^{-\frac{\delta}{2 \gamma} \hat{X}^{2}} \gamma D_{x} e^{\frac{\delta}{2 \gamma} \hat{X}^{2}}
$$

and get the decomposition identity for $e^{-a\left(\gamma D_{x}+\delta \hat{X}\right)^{2}}$

$$
e^{-a\left(\gamma D_{x}+\delta \hat{X}\right)^{2}} \equiv e^{\frac{\gamma}{2 \delta} D_{x}^{2}} e^{-a(\delta \hat{X})^{2}} e^{-\frac{\gamma}{2 \delta} D_{x}^{2}} \equiv e^{-\frac{\delta}{2 \gamma} \hat{X}^{2}} e^{-a\left(\gamma D_{x}\right)^{2}} e^{\frac{\delta}{2 \gamma} \hat{X}^{2}}
$$

which in turn gives rise to the formulae

$$
\begin{aligned}
& e^{-a\left(\gamma D_{x}+\delta \hat{X}\right)^{2}} f(x)=e^{\frac{\gamma}{2 \delta} D_{x}^{2}} e^{-a(\delta x)^{2}} e^{-\frac{\gamma}{2 \delta} D_{x}^{2}} f(x)=e^{-\frac{\delta}{2 \gamma} x^{2}} e^{-a\left(\gamma D_{x}\right)^{2}} e^{\frac{\delta}{2 \gamma} x^{2}} f(x) \\
& e^{-a\left(\gamma D_{x}+\delta \hat{X}\right)^{2}} \delta(x)=e^{\frac{\gamma}{2 \delta} D_{x}^{2}} e^{-a \delta^{2} \hat{X}^{2}} e^{-\frac{\gamma}{2 \delta} D_{x}^{2}} \delta(x)=\frac{1}{\gamma \sqrt{-4 \pi a}} e^{\frac{1-2 a \gamma \delta}{4 a \gamma^{2}} x^{2}}
\end{aligned}
$$

By (32a) then (8a) we get the kernel function the integral transform related to $e^{-a\left(\gamma D_{x}+\delta \hat{X}\right)^{2}}$

$$
\begin{aligned}
e^{-a\left(\gamma D_{x}+\delta \hat{X}\right)^{2}} \delta\left(x-x_{0}\right) & =e^{-a\left(\gamma D_{x}+\delta \hat{X}\right)^{2}} e^{-x_{0} D_{x}} \delta(x)=e^{-x_{0}\left((1+2 a \gamma \delta) D_{x}+2 a \delta^{2} \hat{X}\right)} e^{-a\left(\gamma D_{x}+\delta \hat{X}\right)^{2}} \delta(x) \\
& =e^{\frac{1}{2}(1+2 a \gamma \delta)\left(2 a \delta^{2}\right) x_{0}^{2}} e^{-x_{0} 2 a \delta^{2} x} e^{-x_{0}(1+2 a \gamma \delta) D_{x}} \frac{1}{\gamma \sqrt{-4 \pi a}} e^{\frac{1-2 a \gamma \delta}{4 a \gamma^{2}} x^{2}}
\end{aligned}
$$

so that finally

$$
e^{-a\left(\gamma D_{x}+\delta \hat{X}\right)^{2}} f(x)=\frac{1}{\gamma \sqrt{-4 \pi a}} e^{\frac{1-2 a \gamma \delta}{4 a \gamma^{2}} x^{2}} \int_{-\infty}^{+\infty} e^{\frac{1+2 a \gamma \delta}{4 a \gamma^{2}} x_{0}{ }^{2}} e^{-\frac{1}{2 a \gamma^{2}} x x_{0}} f\left(x_{0}\right) d x_{0}
$$

For the Gauss transform $e^{-a D_{x}^{2}}$, we have $\gamma=1, \delta=0$ so that

$$
e^{-a D_{x}^{2}} f(x)=\frac{1}{\sqrt{-4 \pi a}} \int_{-\infty}^{+\infty} e^{\frac{1}{4 a}\left(x-x_{0}\right)^{2}} f\left(x_{0}\right) d x_{0}
$$




\subsection{The Group of Transforms Realized by $e^{\theta\left(\gamma \hat{X}+\delta D_{x}\right)\left(\alpha \hat{X}+\beta D_{x}\right)}$}

Because

$$
\left[\alpha D_{x}+\beta \hat{X}, \gamma D_{x}+\delta \hat{X}\right] \equiv(\alpha \delta-\beta \gamma) I
$$

we may substitute $\left(D_{x}, \hat{X}\right)$ with $\left(\alpha D_{x}+\beta \hat{X}, \gamma D_{x}+\delta \hat{X}\right)$ where $(\alpha \delta-\beta \gamma)=1$ in the dilatation identities written under the matricial form

$$
e^{\theta \hat{X} D_{x}}\left|\begin{array}{c}
D_{x} \\
\hat{X}
\end{array}\right| e^{-\theta \hat{X} D_{x}} \equiv\left|\begin{array}{rr}
e^{-\theta} & 0 \\
0 & e^{\theta}
\end{array}\right|\left|\begin{array}{r}
D_{x} \\
\hat{X}
\end{array}\right|
$$

and get

$$
\begin{gathered}
e^{\theta\left(\gamma D_{x}+\delta \hat{X}\right)\left(\alpha D_{x}+\beta \hat{X}\right)}\left|\begin{array}{cc}
\alpha & \beta \\
\gamma & \delta
\end{array}\right|\left|\begin{array}{c}
D_{x} \\
\hat{X}
\end{array}\right| e^{-\theta\left(\gamma D_{x}+\delta \hat{X}\right)\left(\alpha D_{x}+\beta \hat{X}\right)} \equiv\left|\begin{array}{cc}
e^{-\theta} & 0 \\
0 & e^{\theta}
\end{array}\right| \begin{array}{cc}
\alpha & \beta \\
\gamma & \delta
\end{array}|| \begin{array}{c}
D_{x} \\
\hat{X}
\end{array} \mid \\
e^{\theta\left(\gamma D_{x}+\delta \hat{X}\right)\left(\alpha D_{x}+\beta \hat{X}\right)}\left|\begin{array}{c}
D_{x} \\
\hat{X}
\end{array}\right| e^{-\theta\left(\gamma D_{x}+\delta \hat{X}\right)\left(\alpha D_{x}+\beta \hat{X}\right)} \equiv\left|\begin{array}{cc}
\alpha \delta e^{-\theta}-\beta \gamma e^{\theta} & -2 \beta \delta \sinh \theta \\
2 \alpha \gamma \sinh \theta & -\beta \gamma e^{-\theta}+\alpha \delta e^{\theta}
\end{array}\right|\left|\begin{array}{c}
D_{x} \\
\hat{X}
\end{array}\right|
\end{gathered}
$$

The above identity gives us the matricial form of the operator $\lambda e^{\theta\left(\gamma \hat{X}+\delta D_{x}\right)\left(\alpha \hat{X}+\beta D_{x}\right)}$ where $\lambda$ is arbitrary

$$
\lambda e^{\theta\left(\gamma \hat{X}+\delta D_{x}\right)\left(\alpha \hat{X}+\beta D_{x}\right)} \cong\left|\begin{array}{cc}
\alpha \delta e^{-\theta}-\beta \gamma e^{\theta} & -2 \beta \delta \sinh \theta \\
2 \alpha \gamma \sinh \theta & -\beta \gamma e^{-\theta}+\alpha \delta e^{\theta}
\end{array}\right|, \forall \lambda \in C
$$

Now in order to calculate the transform of a function $f(x)$ we utilize (38a), (21a), (21b) to firstly get, with $\gamma^{\prime}=\alpha \gamma\left(e^{\theta}-e^{-\theta}\right), \delta^{\prime}=\alpha \delta e^{\theta}-\beta \gamma e^{-\theta}$, the formulae

$$
\begin{aligned}
e^{\theta\left(\gamma D_{x}+\delta \hat{X}\right)\left(\alpha D_{x}+\beta \hat{X}\right)} f(\hat{X}) e^{-\theta\left(\gamma D_{x}+\delta \hat{X}\right)\left(\alpha D_{x}+\beta \hat{X}\right)} & \equiv f\left(\gamma^{\prime} D_{x}+\delta^{\prime} \hat{X}\right) \\
& \equiv e^{-\frac{\delta^{\prime}}{2 \gamma^{\prime}} x^{2}} f\left(\gamma^{\prime} D_{x}\right) e^{\frac{\delta^{\prime}}{2 \gamma^{\prime}} x^{2}} \equiv e^{\frac{\gamma^{\prime}}{2 \delta^{\prime}} D_{x}^{2}} f\left(\delta^{\prime} \hat{X}\right) e^{-\frac{\gamma^{\prime}}{2 \delta^{\prime}} D_{x}{ }^{2}}
\end{aligned}
$$

Acting these identities onto the function $e^{-\frac{\beta}{2 \alpha} x^{2}}$ which has the property $\left(\alpha D_{x}+\beta \hat{X}\right) e^{-\frac{\beta}{2 \alpha} x^{2}}=0$ we then get

$$
\begin{aligned}
& e^{\theta\left(\gamma D_{x}+\delta \hat{X}\right)\left(\alpha D_{x}+\beta \hat{X}\right)} f(x) e^{-\frac{\beta}{2 \alpha} x^{2}}=e^{-\frac{\delta^{\prime}}{2 \gamma^{\prime}} x^{2}} f\left(\gamma^{\prime} D_{x}\right) e^{\frac{\delta^{\prime}}{2 \gamma^{\prime}} x^{2}} e^{-\frac{\beta}{2 \alpha} x^{2}} \\
& e^{\theta\left(\gamma D_{x}+\delta \hat{X}\right)\left(\alpha D_{x}+\beta \hat{X}\right)} f(x) e^{-\frac{\beta}{2 \alpha} x^{2}}=e^{\frac{\gamma^{\prime}}{2 \delta^{\prime}} D_{x}^{2}} f\left(\delta^{\prime} \hat{X}\right) e^{-\frac{\gamma^{\prime}}{2 \delta^{\prime}} D_{x}^{2}} e^{-\frac{\beta}{2 \alpha} x^{2}}=\left(\frac{e^{\theta}}{\delta^{\prime}}\right)^{-\frac{1}{2}} e^{\frac{\gamma^{\prime}}{2 \delta^{\prime} D_{x}^{2}}} f\left(\delta^{\prime} \hat{X}\right) e^{-\frac{\beta \delta}{2 \alpha} e^{-\theta} x^{2}}
\end{aligned}
$$

Replacing $f(x)$ with $f(x) e^{\frac{\beta}{2 \alpha} x^{2}}$ we get finally

$$
\begin{aligned}
& e^{\theta\left(\gamma D_{x}+\delta \hat{X}\right)\left(\alpha D_{x}+\beta \hat{X}\right)} f(x)=e^{-\frac{\delta^{\prime}}{2 \gamma^{\prime}} x^{2}} f\left(\gamma^{\prime} D_{x}\right) e^{\frac{\beta}{2 \alpha}\left(\gamma D_{x}{ }^{2}\right.} e^{\frac{\delta^{\prime}}{2 \gamma^{\prime}} x^{2}} e^{-\frac{\beta}{2 \alpha} x^{2}} \\
& e^{\theta\left(\gamma D_{x}+\delta \hat{X}\right)\left(\alpha D_{x}+\beta \hat{X}\right)} f(x)=e^{\frac{\gamma^{\prime}}{2 \delta^{\prime} D_{x}^{2}}} f\left(\delta^{\prime} x\right) e^{\frac{\beta}{2 \alpha}\left(\delta x^{2}\right.} e^{-\frac{\theta}{2}} \sqrt{\delta} e^{-\frac{\beta \delta}{2 \alpha} e^{-\theta} x^{2}} \\
& =e^{-\frac{\theta}{2}} \sqrt{\delta^{\prime}} e^{\frac{\gamma^{\prime}}{2 \delta^{\prime}} D_{x}^{2}} f\left(\delta^{\prime} x\right) e^{\beta \delta \delta^{\prime} \sinh \theta x^{2}}=e^{-\frac{\theta}{2}} \sqrt{\delta} e^{\frac{\gamma^{\prime}}{2 \delta^{\prime} D_{x}^{2}}} e^{\ln \delta \hat{X} D_{x}} e^{\frac{\beta \delta}{\delta} \sinh \theta x^{2}} f x \\
& e^{\theta\left(\gamma D_{x}+\delta \hat{X}\right)\left(\alpha D_{x}+\beta \hat{X}\right)} \delta(x)=e^{-\frac{\theta}{2}} \frac{1}{\sqrt{2 \pi \gamma^{\prime}}} e^{-\frac{\delta^{\prime}}{2 \gamma^{\prime}} x^{2}} \text { with } \gamma^{\prime}=\alpha \gamma\left(e^{\theta}-e^{-\theta}\right), \delta^{\prime}=\alpha \delta e^{\theta}-\beta \gamma e^{-\theta}
\end{aligned}
$$

The transform of $\delta\left(x-x_{0}\right)$ is straightforward from (38a), (38e) and (8a). 
The formula (38d) leads to the decomposition identity for $e^{\theta\left(\gamma D_{x}+\delta \hat{X}\right)\left(\alpha D_{x}+\beta \hat{X}\right)}$

$$
e^{\theta\left(\gamma D_{x}+\delta \hat{X}\right)\left(\alpha D_{x}+\beta \hat{X}\right)} \equiv e^{-\frac{\theta}{2}} \sqrt{\Delta} e^{\frac{\Gamma}{2 \Delta} D_{x}^{2}} e^{\ln \Delta \hat{X} D_{x}} e^{\frac{\beta \delta}{\Delta} \sinh \theta \hat{X}^{2}}
$$

where we define

$$
\Gamma=\alpha \gamma\left(e^{\theta}-e^{-\theta}\right), \Delta=\alpha \delta e^{\theta}-\beta \gamma e^{-\theta}
$$

Now, let us utilized $(39 \mathrm{a}, 39 \mathrm{~b})$ to calculate the product

$$
e^{\theta^{\prime \prime}\left(\gamma^{\prime \prime} \hat{X}+\delta^{\prime \prime} D_{x}\right)\left(\alpha^{\prime \prime} \hat{X}+\beta^{\prime \prime} D_{x}\right)} \equiv e^{\theta^{\prime}\left(\gamma^{\prime} \hat{X}+\delta^{\prime} D_{x}\right)\left(\alpha^{\prime} \hat{X}+\beta^{\prime} D_{x}\right)} e^{\theta\left(\gamma \hat{X}+\delta D_{x}\right)\left(\alpha \hat{X}+\beta D_{x}\right)}
$$

Firstly by utilizing (39a) and (21b) we can write

$$
\begin{aligned}
e^{\theta^{\prime}\left(\gamma^{\prime} D_{x}+\delta^{\prime} \hat{X}\right)\left(\alpha^{\prime} D_{x}+\beta^{\prime} \hat{X}\right)} e^{\theta\left(\gamma D_{x}+\delta \hat{X}\right)\left(\alpha D_{x}+\beta \hat{X}\right)} & \equiv e^{-\frac{\theta}{2}} \sqrt{\Delta} e^{\theta^{\prime}\left(\gamma^{\prime} D_{x}+\delta^{\prime} \hat{X}\right)\left(\alpha^{\prime} D_{x}+\beta^{\prime} \hat{X}\right)} e^{\frac{\Gamma}{2 \Delta} D_{x}^{2}} e^{\ln \Delta \hat{X} D_{x}} e^{\frac{\beta \delta}{\Delta} \sinh \theta \hat{X}^{2}} \\
& \equiv e^{-\frac{\theta}{2}} \sqrt{\Delta} e^{\frac{\Gamma}{2 \Delta} D_{x}^{2}} e^{\theta^{\prime}\left(\gamma^{\prime} D_{x}+\delta^{\prime}\left(\hat{X}-\frac{\Gamma}{\Delta} D_{x}\right)\right)\left(\alpha^{\prime} D_{x}+\beta^{\prime}\left(\hat{X}-\frac{\Gamma}{\Delta} D_{x}\right)\right)} e^{\ln \Delta \hat{X} D_{x}} e^{\frac{\beta \delta}{\Delta} \sinh \theta \hat{X}^{2}}
\end{aligned}
$$

Putting

$$
\begin{aligned}
& \Gamma^{\prime}=\left(\alpha^{\prime}-\beta^{\prime} \Gamma / \Delta\right)\left(\gamma^{\prime}-\delta^{\prime} \Gamma / \Delta\right)\left(e^{\theta^{\prime}}-e^{-\theta^{\prime}}\right) \\
& \Delta^{\prime}=\left(\alpha^{\prime} \delta^{\prime}-\beta^{\prime} \delta^{\prime} \Gamma / \Delta\right) e^{\theta^{\prime}}-\left(\beta^{\prime} \gamma^{\prime}-\beta^{\prime} \delta^{\prime} \Gamma / \Delta\right) e^{-\theta^{\prime}}
\end{aligned}
$$

we get, utilizing (13a),

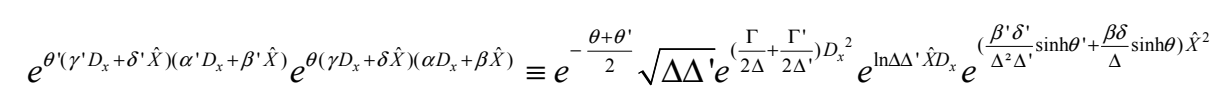

Finally we may define $\theta^{\prime \prime}, \Delta ", \Gamma ", \beta " \delta^{\prime \prime}$ as follows

$$
\theta^{\prime \prime}=\theta+\theta^{\prime}, \Delta^{\prime \prime}=\Delta \Delta^{\prime}, \frac{\Gamma^{\prime \prime}}{\Delta^{\prime \prime}}=\frac{\Gamma}{\Delta}+\frac{\Gamma^{\prime}}{\Delta^{\prime}}, \frac{\beta^{\prime \prime} \delta^{\prime \prime} \sinh \theta^{\prime \prime}}{\Delta^{\prime \prime}}=\frac{\beta^{\prime} \delta^{\prime} \sinh \theta^{\prime}}{\Delta \Delta}+\frac{\beta \delta \sinh \theta}{\Delta}
$$

and get

$$
e^{\theta^{\prime \prime}\left(\gamma^{\prime \prime} \hat{X}+\delta^{\prime \prime} D_{x}\right)\left(\alpha^{\prime \prime} \hat{X}+\beta^{\prime \prime} D_{x}\right)} \equiv e^{-\frac{\theta^{\prime \prime}}{2}} \sqrt{\Delta "} e^{\frac{\Gamma^{\prime \prime}}{2 \Delta^{\prime \prime}} D_{x}^{2}} e^{\ln \Delta^{\prime \prime} \hat{X} D_{x}} e^{\frac{\beta^{\prime \prime} \delta^{\prime \prime}}{\Delta^{\prime \prime}} \sinh \theta^{\prime \prime} \hat{X}^{2}}
$$

As conclusion we see that the differential transforms realized by the operators $e^{\theta\left(\gamma \hat{X}+\delta D_{x}\right)\left(\alpha \hat{X}+\beta D_{x}\right)}$ form a group. This group contains the dilatation, the Baker-Campbell-Hausdorff, Bargmann, Fourier, bilateral Laplace transforms and the subgroup of fractional order Fourier transforms that we will study hereinafter.

For example, with $\alpha=-\delta=-i / \sqrt{2}, \beta=-\gamma=1 / \sqrt{2}$ we have

$$
\begin{aligned}
& e^{i \theta} e^{\theta\left(D_{x}^{2}+\hat{X}^{2}\right)} \equiv e^{-i 2 \theta \frac{\left(-D_{x}+i \hat{X}\right)\left(-i D_{x}+\hat{X}\right)}{2}} \cong\left|\begin{array}{cc}
\cos 2 \theta & -\sin 2 \theta \\
\sin 2 \theta & \cos 2 \theta
\end{array}\right| \\
& e^{i \theta} e^{\theta\left(D_{x}^{2}+\hat{X}^{2}\right)} \equiv e^{-i 2 \theta \frac{\left(-D_{x}+i \hat{X}\right)\left(-i D_{x}+\hat{X}\right)}{2}} \equiv e^{i \theta} \sqrt{\cos 2 \theta} e^{\frac{1}{2} \operatorname{tg} 2 \theta D_{x}^{2}} e^{\ln \cos 2 \theta \hat{X} D_{x}} e^{\frac{1}{2} \operatorname{tg} 2 \theta x^{2}}
\end{aligned}
$$

This identity is equivalent to the Baker-Campbell-Hausdorff relation obtained by multiplication of matrices representing operators $e^{\frac{1}{2} \operatorname{tg} 2 \theta D_{x}^{2}}, e^{\ln \cos 2 \theta \hat{X} D_{x}}, e^{\frac{1}{2} \operatorname{tg} 2 \theta x^{2}}$ quoted in Wolf (1979).

From this relation and (28) we get the formulae

$$
e^{\theta\left(\hat{X}^{2}+D_{x}^{2}\right)} f(x)=\sqrt{\cos 2 \theta} e^{\frac{1}{2} \operatorname{tg} 2 \theta D_{x}^{2}} e^{\frac{\sin 4 \theta}{4} x^{2}} f(x \cos 2 \theta)
$$




$$
\begin{aligned}
& e^{\theta\left(\hat{X}^{2}+D_{x}^{2}\right)} \delta(x)=\sqrt{\cos 2 \theta} e^{\frac{1}{2} \operatorname{tg} 2 \theta D_{x}^{2}} e^{\frac{\sin 4 \theta}{4} x^{2}} \delta(x \cos 2 \theta)=\frac{\sqrt{\cos 2 \theta}}{|\cos 2 \theta| \sqrt{2 \pi \operatorname{tg} 2 \theta}} e^{-\frac{1}{2 \operatorname{tg} 2 \theta} x^{2}} \\
& e^{\theta\left(\hat{X}^{2}+D_{x}^{2}\right)} \delta\left(x-x_{0}\right)=e^{\theta\left(\hat{X}^{2}+D_{x}^{2}\right)} e^{-x_{0} D_{x}} \delta(x)=e^{-x_{0}\left(\cos 2 \theta D_{x}-\sin 2 \theta \hat{X}\right)} \frac{1}{\sqrt{2 \pi \sin 2 \theta}} e^{-\frac{1}{2 \operatorname{tg} 2 \theta} x^{2}}
\end{aligned}
$$

The integral transform corresponding to the Baker-Campbell-Hausdorff transform is then obtainable using (8a) and (24). The Bargmann transform corresponds to the case where $\theta=\pi / 8$ (Wolf, 1979).

Equations (41c), (41d), (41d) may be utilized to get the Green's function of $e^{-\theta\left(\hat{X}^{2}+D_{x}^{2}\right)}$, obtain the kernel of its related integral transform and resolve the Schrödinger equation corresponding to a suitable second order potential.

\subsection{The Fractional Order Fourier Transforms}

Consider the operator $F T^{(\theta)} \equiv e^{i \theta} e^{i \theta \frac{\left(D_{x}+\hat{X}\right)\left(D_{x}-\hat{X}\right)}{2}}$ corresponding to $e^{i \theta} e^{i \theta\left(\gamma \hat{X}+\delta D_{x}\right)\left(\alpha \hat{X}+\beta D_{x}\right)}$ with $\alpha=\delta=\gamma=-\beta=1 / \sqrt{2}$. According to $(38 \mathrm{~b})$ we have

$$
F T^{(\theta)} \equiv e^{i \theta} e^{i \theta \frac{\left(D_{x}+\hat{X}\right)\left(D_{x}-\hat{X}\right)}{2}} \cong\left|\begin{array}{ll}
\cos \theta & i \sin \theta \\
i \sin \theta & \cos \theta
\end{array}\right|
$$

From the decomposition identity (39a, 39b) we get

$$
\begin{aligned}
& F T^{(\theta)} f(x)=e^{i \theta} e^{i \theta \frac{\left(D_{x}+\hat{X}\right)\left(D_{x}-\hat{X}\right)}{2}} f(x)=e^{i \frac{\theta}{2}} \sqrt{\cos \theta} e^{\frac{i}{2} \operatorname{tg} \theta D_{x}^{2}} e^{\ln \cos \theta \hat{X} D_{x}} e^{-\frac{i}{2} \operatorname{tg} \theta \hat{X}^{2}} f(x) \\
& =e^{i \frac{\theta}{2}} \sqrt{\cos \theta} e^{\frac{i}{2} \operatorname{tg} \theta D_{x}^{2}} e^{-\frac{i}{4} \sin 2 \theta x^{2}} f(\cos \theta x) \\
& F T^{(\theta)} \delta(x)=e^{i \frac{\theta}{2}} \sqrt{\cos \theta} e^{\frac{i}{2} \operatorname{tg} \theta D_{x}^{2}} \frac{1}{|\cos \theta|} \delta(x)=e^{i \frac{\theta}{2}} \frac{1}{\sqrt{2 \pi i \sin \theta}} e^{\frac{i}{2} \operatorname{cotg} \theta x^{2}} \delta(x)
\end{aligned}
$$

The above formula gives the Green's function of $F T^{(-\theta)}$.

In order to obtain the integral transform form related to $F T^{(\theta)}$ let us use (8a) to calculate firstly the kernel

$$
\begin{aligned}
& F T^{(\theta)} \delta\left(x-x_{0}\right)=F T^{(\theta)} e^{-x_{0} D_{x}} \delta(x)=e^{-x_{0}\left(\cos \theta D_{x}+i \sin \theta \hat{X}\right)} F T^{(\theta)} \delta(x) \\
& =e^{i \frac{\theta}{2}} \frac{1}{\sqrt{2 \pi i \sin \theta}} e^{-x_{0}\left(\cos \theta D_{x}+i \sin \theta \hat{X}\right)} e^{\frac{i}{2} \cot \theta \theta x^{2}} \\
& =e^{i \frac{\theta}{2}} e^{-i \frac{\pi}{4}+i n \pi} \frac{1}{\sqrt{2 \pi \sin \theta}} e^{\frac{i}{2} \operatorname{cotg} \theta x_{0}{ }^{2}} e^{-\frac{i x x_{0}}{\sin \theta}} e^{\frac{i}{2} \cot \theta \theta x^{2}}, n \in Z
\end{aligned}
$$

Finally according to (24) we get

$$
F T^{(\theta)} f(x)=e^{i \frac{\theta}{2}-i \frac{\pi}{4}+i n \pi} \frac{1}{\sqrt{2 \pi \sin \theta}} e^{\frac{i}{2} \operatorname{cotg} \theta x^{2}} \int_{-\infty}^{+\infty} e^{\frac{i}{2} \operatorname{cotg} \theta x_{0}{ }^{2}} e^{-\frac{i x x_{0}}{\sin \theta}} f\left(x_{0}\right) d x_{0}, n \in Z
$$

This formula is to be compared with the formulae of Namias V. and of McBride A.C. and Kerr F.H. given in the Transforms and Applications Handbook (Poularikas, 2000).

\section{The Fourier Transform}

\subsection{Representative Differential Operators}

Consider the integral representation of the operator $F T^{\left(\frac{\pi}{2}\right)}$. According to (42e) 


$$
F T^{\left(\frac{\pi}{2}\right)} f(x)=e^{i n \pi} \frac{1}{\sqrt{2 \pi}} \int_{-\infty}^{+\infty} e^{-\frac{i x x_{0}}{1}} f\left(x_{0}\right) d x_{0}
$$

so that with the choice $\mathrm{n}$ pair we get

$$
F T \equiv F T^{\left(\frac{\pi}{2}\right)} \equiv e^{i \frac{\pi}{2}} e^{i \frac{\pi}{4}\left(D_{x}+\hat{X}\right)\left(D_{x}-\hat{X}\right)} \equiv e^{i \frac{\pi}{4}} e^{i \frac{\pi}{4}\left(D_{x}^{2}-\hat{X}^{2}\right)}
$$

in accordance with the result obtained by Wolf K.B. when considering harmonic oscillator wave functions (Wolf, 1979).

The Fourier transform must transforms $\left(D_{x}, \hat{X}\right)$ into $\left(i \hat{X}, i D_{x}\right)$ so that it can't be decomposed according to (39a). Instead we may utilized (21a), (21b) to get

$$
\begin{aligned}
& e^{c X^{2}} e^{b D_{x}^{2}} e^{a X^{2}}\left|\begin{array}{c}
D_{x} \\
\hat{X}
\end{array}\right| e^{-a X^{2}} e^{-b D_{x}^{2}} e^{-c X^{2}} \equiv\left|\begin{array}{cc}
1-4 a b & 8 a b c-2 a-2 c \\
2 b & 1-4 b c
\end{array}\right|\left|\begin{array}{c}
D_{x} \\
\hat{X}
\end{array}\right| \\
& e^{-\frac{i}{2} X^{2}} e^{\frac{i}{2} D_{x}^{2}} e^{-\frac{i}{2} X^{2}}\left|\begin{array}{c}
D_{x} \\
\hat{X}
\end{array}\right| e^{\frac{i}{2} X^{2}} e^{-\frac{i}{2} D_{x}^{2}} e^{\frac{i}{2} X^{2}} \equiv\left|\begin{array}{cc}
0 & i \\
i & 0
\end{array}\right|\left|\begin{array}{c}
D_{x} \\
\hat{X}
\end{array}\right|
\end{aligned}
$$

so that

$$
F T \equiv \lambda e^{-\frac{i}{2} \hat{X}^{2}} e^{\frac{i}{2} D_{x}^{2}} e^{-\frac{i}{2} \hat{X}^{2}}
$$

The factor $\lambda$ has to be chosen according to the conventional definition ${ }_{1}(23)$ of FT.

As this conventional definition implies (27), (28) and $F T \delta(x)=(2 \pi)^{-\frac{1}{2}}$ we get

$$
F T \delta(x)=\lambda e^{-\frac{i}{2} \hat{X}^{2}} e^{\frac{i}{2} D_{x}^{2}} e^{-\frac{i}{2} \hat{X}^{2}} \delta(x)=\lambda e^{-\frac{i}{2} \hat{X}^{2}} e^{\frac{i}{2} D_{x}^{2}} \delta(x)=\lambda e^{-\frac{i}{2} \hat{X}^{2}}(2 \pi i)^{-\frac{1}{2}} e^{-\frac{i}{2 i} x^{2}}=(2 \pi)^{-\frac{1}{2}}
$$

This gives $\lambda=\sqrt{i}$ and

$$
F T \equiv e^{\frac{i \pi}{4}} e^{-\frac{i}{2} \hat{X}^{2}} e^{\frac{i}{2} D_{x}^{2}} e^{-\frac{i}{2} \hat{X}^{2}}
$$

Moreover, taking the Fourier transform itself of both members of (45) we get also

$$
F T \equiv e^{\frac{i \pi}{4}} e^{\frac{i}{2} D_{x}^{2}} e^{-\frac{i}{2} \hat{X}^{2}} e^{\frac{i}{2} D_{x}^{2}}
$$

\subsection{Properties of the Fourier Transform}

Hereinafter we write down the principal properties of the Fourier transform in a concise but comprehensive manner utilizing its differential representation.

Utilizing the conventional definition of the Fourier transform in the study the Gaussian transform we have had

$$
\text { ○ } F T 1=\sqrt{2 \pi} \delta(x), F T \delta(x)=(2 \pi)^{-\frac{1}{2}}, F T e^{-\frac{1}{2} x^{2}}=e^{-\frac{1}{2} x^{2}}
$$

From this we get a very useful formula (Wolf, 1979; Si, 1979)

$$
\text { ○ } \quad \operatorname{FTf}(x)=\operatorname{FTf}(\hat{X}) 1=f\left(i D_{x}\right) F T 1=\sqrt{2 \pi} f\left(i D_{x}\right) \delta(x)
$$

which leads to

$$
\begin{aligned}
& \circ \quad \operatorname{FTFTf}(x)=F T f\left(i D_{x}\right) F T 1=f\left(i^{2} x\right) F T F T 1=f(-x) \\
& \circ \quad F T f^{*}(x)=f^{*}\left(i D_{x}\right) \delta(x)=\left(f\left(-i D_{x}\right) \delta(x)\right)^{*}=(F T f(-x))^{*}
\end{aligned}
$$

and the famous formula involving a convolution product 


$$
\begin{aligned}
\circ \operatorname{FTf}(x) g(x) & =f\left(i D_{x}\right) G(x)=f\left(i D_{x}\right) \int_{-\infty}^{+\infty} G\left(x_{0}\right) \delta\left(x-x_{0}\right) d x_{0} \\
& =\sqrt{2 \pi} \int_{-\infty}^{+\infty} G\left(x_{0}\right) F\left(x-x_{0}\right) d x_{0}=\sqrt{2 \pi} F(x) * G(x)
\end{aligned}
$$

Formula (48) may be use to transform and eventually resolve the Fredholm equation (Si, 1979)

○ $\mu \Psi(z)=\lambda \int_{-\infty}^{+\infty} \Psi\left(z_{0}\right) K\left(z-z_{0}\right) d z_{0}+\Phi(z)=\lambda k\left(i D_{x}\right) \Psi(z)+\Phi(z)$

$$
\Psi(z)=\frac{1}{\mu-\lambda k\left(i D_{x}\right)} \varphi(z) \text { where } k(z)=F T^{-1} K(z)
$$

Combination of FT with the translation and dilatation transforms gives

$$
\begin{aligned}
& \text { ○ } \quad F T f(x+a)=F T e^{a D_{x}} f(x)=e^{i a x} F T f(x)=e^{i a x} F(x) \text { where } F(x)=F T f(x) \\
& \text { ○ } \quad F T e^{i a x} f(x)=e^{-a D_{x}} F T f(x)=F(x-a) \\
& \text { ○ } \operatorname{FTf}\left(e^{a} x\right)=F \operatorname{TT} e^{a \hat{X} D_{x}} f(x)=e^{-a D_{x} \hat{X}} F T f(x)=e^{-a \hat{X} D_{x}-a} F(x)=e^{-a} F\left(e^{-a} x\right) \\
& F T f\left(-e^{a} x\right)=F T e^{a \hat{X} D_{x}} f(-x)=e^{-a} e^{-a \hat{X} D_{x}} \operatorname{FTf}(-x)=e^{-a} e^{-a \hat{X} D_{x}} F T^{-1} f(x)
\end{aligned}
$$

Only when $\mathrm{f}(\mathrm{x})$ is pair that $F T f(\lambda x)=|\lambda|^{-1} F\left(\lambda^{-1} x\right)$

From the property of the Dirac delta function

$$
\frac{1}{\sqrt{2 \pi}} \int_{-\infty}^{+\infty} e^{-i\left(-x_{0}+y_{0}+z_{0}\right) x} d x=\sqrt{2 \pi} \delta\left(-x_{0}+y_{0}+z_{0}\right)
$$

we get a generalization of the Parseval formula

$$
\begin{gathered}
F^{*}(x) G(x) H(x)=(2 \pi)^{-\frac{3}{2}} \int_{-\infty}^{+\infty} \int_{-\infty}^{+\infty} \int_{-\infty}^{+\infty} e^{-i\left(-x_{0}+y_{0}+z_{0}\right) x} f^{*}\left(x_{0}\right) g\left(y_{0}\right) h\left(z_{0}\right) d x_{0} d y_{0} d z_{0} \\
\int_{-\infty}^{+\infty} F^{*}(x) G(x) H(x) d x=(2 \pi)^{-\frac{1}{2}} \int_{-\infty}^{+\infty} \int_{-\infty}^{+\infty} \int_{-\infty}^{+\infty} \delta\left(-x_{0}+y_{0}+z_{0}\right) f^{*}\left(x_{0}\right) g\left(y_{0}\right) h\left(z_{0}\right) d x_{0} d y_{0} d z_{0} \\
=(2 \pi)^{-\frac{1}{2}} \int_{-\infty}^{+\infty} \int_{-\infty}^{+\infty} f^{*}\left(y_{0}+z_{0}\right) g\left(y_{0}\right) h\left(z_{0}\right) d y_{0} d z_{0} \\
=(2 \pi)^{-\frac{1}{2}} \int_{-\infty}^{+\infty} \int_{-\infty}^{+\infty} f^{*}(x+y) g(x) h(y) d x d y
\end{gathered}
$$

The Parseval formula corresponds to $H(x)=1$ and $h(y)=\sqrt{2 \pi} \delta(y)$.

- The calculations of the Fourier transforms of Gaussian functions, of functions related to $\delta(x)$ are straightforward from the differential representation (45), (46) of FT together with the formulae (27), concerning the Gaussian transform.

From (21a), (29), (43) we have

$$
\begin{aligned}
& e^{-\frac{1}{2} \hat{X}^{2}}\left(D_{x}^{2}-2 \hat{X} D_{x}+2 n\right) H_{n}(x)=0 \\
& \left.\left(D_{x}^{2}-\hat{X}^{2}+2 n+1\right) e^{-\frac{1}{2} \hat{X}^{2}} H_{n}(x)=0\right)
\end{aligned}
$$

and see that $e^{-\frac{1}{2} \hat{X}^{2}} H_{n}(x)$ is the solution of the harmonic oscillator differential equation and at the same time the eigenfunction of $F T^{(\theta)}$ and consequently of FT 


$$
\circ \quad F T^{(\theta)} e^{-\frac{1}{2} x^{2}} H_{n}(x)=e^{i \frac{\theta}{2}} e^{-i \frac{\theta}{2}(2 n+1)} e^{-\frac{1}{2} x^{2}} H_{n}(x)
$$

4.3 The Fourier Transform in 3D Space

In $3 \mathrm{D}$ space we may define the differential by using the operator gradient $\nabla$, say

$$
\circ \quad F(\vec{r})=F T f(\vec{r})=(2 \pi)^{-\frac{3}{2}} f(i \nabla) \delta(\vec{r})
$$

In a change of the argument $\vec{r}$ into $U \vec{r}$ where $\mathrm{U}$ is an reversible matrix, $\nabla$ is changed into $\tilde{U}^{-1} \nabla$, so that let $V \equiv \tilde{U}^{-1}$ we get

$$
\begin{aligned}
\circ \quad \operatorname{FTf}(U \vec{r}) & =(2 \pi)^{-\frac{3}{2}} f\left(i \tilde{U}^{-1} \nabla\right) \delta(U \vec{r})=(2 \pi)^{-\frac{3}{2}}|\operatorname{det} U|^{-1} f\left(i \tilde{U}^{-1} \nabla\right) \delta(\vec{r}) \\
& =|\operatorname{det} U|^{-1} F T f(V \vec{r})
\end{aligned}
$$

Now let $\left(\vec{u}_{1}, \vec{u}_{2}, \vec{u}_{3}\right)$ be three line vectors extracted from $\mathrm{U}$ and $\left(\vec{v}_{1}, \vec{v}_{2}, \vec{v}_{3}\right)$ the line vectors extracted from V. Because $U V \tilde{V} \equiv I$ we see that $\left(\vec{u}_{1}, \vec{u}_{2}, \vec{u}_{3}\right)$ and $\left(\vec{v}_{1}, \vec{v}_{2}, \vec{v}_{3}\right)$ are mutually reciprocal

$$
\begin{aligned}
& \vec{u}_{i} \vec{v}_{j}=\delta_{i j} \\
& \vec{r}=\sum_{i=1}^{3}\left(\vec{v}_{i} \vec{r}\right) \vec{u}_{i}=\sum_{i=1}^{3}\left(\vec{u}_{i} \vec{r}\right) \vec{v}_{i}
\end{aligned}
$$

The formula (59) in turn is crucial for finding the Fourier transform of a geometric form delimited by planes as shown in the following example

- Let $\mathrm{P}$ be the plane defined by two vectors $\left(\vec{u}_{2}, \vec{u}_{3}\right)$ in the system of coordinates scanned

by $\left(\vec{u}_{1}, \vec{u}_{2}, \vec{u}_{3}\right)$. As $\mathrm{P}$ contains only points having oblique component equal to zero on the axis $\vec{u}_{1}$ it is represented by the function

$$
f(\vec{r})=\delta\left(\vec{v}_{1} \vec{r}\right) u\left(\vec{v}_{2} \vec{r}\right) u\left(\vec{v}_{3} \vec{r}\right), u(x)=1
$$

The Fourier transform of $\mathrm{P}$

$$
F T f(\vec{r})=|\operatorname{det} U| u\left(\vec{v}_{1} \vec{r}\right) \delta\left(\vec{v}_{2} \vec{r}\right) \delta\left(\vec{v}_{3} \vec{r}\right)
$$

describes, by (60), a geometric form having components zero on $\vec{u}_{2}$ and $\vec{u}_{3}$, i.e. the system of straight lines perpendicular to $P$.

More examples may be found in $\mathrm{Si}$ (2014) where the Fourier transform is utilized to get the amplitudes of plane waves diffracted by a planar interface or an oblique pyramid.

\section{Remarks and Conclusion}

The main contribution of this work consists in proving that from and only from the definitions of the "multiplication by the argument" operator $\hat{X}$ by Eckart, the Fourier integral transform and the Dirac delta function $\delta(x)$, one can obtain the transforms of operators and functions by the translation operator $e^{a D_{x}}$ and the Gaussian operator $e^{a D_{x}^{2}}$; then that all the relations between operators issued from these transforms are invariant under substituting the couple of operators $\left(D_{x}, \hat{X}\right)$ with any other couple $(A, B)$ provided that $A B-B A \equiv I$.

As applications we have studied with success the transformational properties of the transforms realized by the group of operators $e^{a\left(\alpha D_{x}+\beta \hat{X}\right)}$, of the operators $e^{a \hat{X}^{2} D_{x}}, e^{\ln a \ln \ln \hat{X}_{x}}, e^{u(\hat{X}) D_{x}}, e^{a\left(\alpha D_{x}+\beta \hat{X}\right)^{2}}$ and of the group of operators $e^{a\left(\alpha D_{x}+\beta \hat{X}\right)\left(\gamma D_{x}+\delta \hat{X}\right)}$ which contains the dilatation, the Bargmann, etc... transforms, the fractional order Fourier transforms and the Fourier transform itself.

The second remark is that one may decompose these differential transforms into product of translation, dilatation and Gaussian ones and by this way obtain the integral transform corresponding to a differential transform. Applications of differential transforms in science and engineering are thus assured. Applications in the domains of differential equations and analytic geometry are also possible.

The third remark is that by this method we may obtain easily quasi all the properties of the Fourier transform without using the cumbersome technique of integral calculations. 
The last but not least remark is that this work may be utilized in quantum mechanics by replacing

$$
D_{x} \text { by } \hat{p} \equiv-i \hbar D_{x} \text { and } \hat{X} \text { by } \hat{q}, a^{+} \equiv \frac{1}{\sqrt{2}}\left(D_{x}-\hat{X}\right), a \equiv \frac{1}{\sqrt{2}}\left(D_{x}+\hat{X}\right) \text {. }
$$

We wish that the definition of the omnipresent integral Fourier transform which is the egg of Gaussian then of the differential Fourier transform among others in this work would be standardized to avoid discordance when applying it in science and engineering.

We hope that would have a new version of MS Word where numerical calculations and graphic drawing are possible.

Finally we wish that there would have students and researchers who continue to apply and develop the method in this work to study the calculus in quantum mechanics, other integral transforms and to extend it to couples of dual operators such as $\left(D_{x}+u(\hat{X}), \hat{X}\right)$ and to $\mathrm{N}$-dimensional spaces.

\section{Acknowledgments}

The author dedicates this work in memory to Prof. Tu Ngoc Tinh for the formation in mathematics he had given him at the Dalat University in Vietnam about fifty years ago. He would like to thank very much Prof. Wolf K.B. who had offered him his book on Integral transforms in 1980 when he was an anonymous researcher at the Université de l'Etat à Mons in Belgium. This book has suggested him to think about the problem frequently. Many thanks also for Prof. C. Quesne for interesting him canonical transform at the Université libre de Bruxelles.

Lastly, he feels that never he can forget the jasmine tea his wife brought to him from time to time during the long months he persevered for accomplishing this work.

\section{Reference}

Abramovitz, M., \& Stegun, I. A. (1968). Handbook of Mathematical Functions. New York: Dover.

Si, D. T. (1979). Resolution of Fredholm equations with kernels K (z- $\left.z_{0}\right)$ by operational calculus. Journal of Mathematical Physics, 20, 1306-1307. http://dx.doi.org/10.1063/1.524231

Si, D. T. (2014). On amplitude of Fraunhofer diffractions of waves by 3D objects. J. Opt, 43(3), 219-230. http://dx.doi.org/10.1007/s12596-014-0220-0

Eckart, C. (1926). Operator calculus and the solutions of the equations of quantum dynamics. Phys. Rev., 28, 711-26. http://dx.doi.org/10.1103/PhysRev.28.711

Oldham, K. B., \& Spanier, J. (1974). The fractional calculus. New York, London: Academic Press.

Poularikas, A. D. (2000). The Transforms and Applications Handbook (2nd ed.). CRC Press LLC.

Erdélyi, A., Magnus, W., Oberhettinger, F., \& Tricomi, F. G. (1962). Tables of higher Transcendental Functions and Tables of Integral transforms. New York: McGraw-Hill.

Liouville, J. (1832). Mémoire sur l'intégration de l'équation $\left(m x^{2}+n x+p\right) d^{2} / d x^{2}+\left(a_{1}+b_{1} x\right) d / d x+a_{0} y=0$. à l'aide des différentielles à indices quelconques. J. École Polytechnique, 13, 163-186.

Wilcox, R. M. (1967). Exponential operators and parameters differentiation in quantum physics. J. Mathematical Phys., 8, 962-982. http://dx.doi.org/10.1063/1.1705306

Wolf, K. B. (1976). Hyperdifferential operator s and integral transforms. Rev. Mexicana Fis., March.

Wolf, K. B. (1979). Integral transforms in Science and Engineering. New York: Plenum Press. http://dx.doi.org/10.1007/978-1-4757-0872-1

Dirac, P. A. M. (1930). Quantum Mechanics (4th ed., pp 1-97). London: Oxford Univ. Press.

\section{Copyrights}

Copyright for this article is retained by the author(s), with first publication rights granted to the journal.

This is an open-access article distributed under the terms and conditions of the Creative Commons Attribution license (http://creativecommons.org/licenses/by/3.0/). 